\title{
Behaviour of Iron Ion in the Morphosynthesis of Magnetite Particles
}

\author{
Jui Chakraborty, Swapan Kumar Das, V. Rao and Arvind Sinha \\ National Metallurgical Laboratory, Jamshedpur-831007, India
}

In situ synthesis of magnetite particles has been carried out in a preorganized polyvinyl alcohol (PVA) gel by varying the total number of iron ions in an environment of a fixed number of hydroxyl functional groups of PVA. A systematic change in the morphology and patterning of the magnetite particles on the polymer surface revealed the effect of the total number of cations on the conformation of the supramolecular matrix, responsible for dictating the morphological features of the magnetite particles.

(Received September 6, 2002; Accepted April 14, 2003)

Keywords: biomimetic synthesis, magnetite particles, polyvinyl alcohol

\section{Introduction}

With a high degree of uniformity in the size, morphology and orientation, nanosized iron oxide particles find application in diverse technological fields ranging from pharmaceuticals to magnetic memory storage. However, the magnetite particles produced synthetically or formed extra-cellularly by the metabolic activities of some iron-reducing bacteria, are not fully crystallized and exhibit no control over the particles dimension and their morphological features. The synthesis of fine sized inorganic particles exhibiting highly ordered structures has been intensively explored in recent years. ${ }^{1)}$ Biomimetic synthesis, a novel strategy akin to organic-matrix mediated biomineralization process, has been recently established to produce advanced materials with highly ordered structures exhibiting a better structure-property correlation. $^{2-5)}$ Analogous to the enzymatically controlled in situ mineralisation of biomaterials, different self assembled bio- and synthetic polymer matrix characterized by highly acidic functional groups have felicitated the growth of different nanocrystalline materials in general and oxides in particular. ${ }^{6-9)}$ Instead of using a supramolecular matrix comprising highly acidic functional groups known for chemical chelation, Sinha et al. have recently demonstrated that a simple polymer like PVA having polar hydroxyl functional groups can act as a supramolecular matrix for the synthesis of nanosized metal oxides and bioceramic particles. ${ }^{10)}$ The present work demonstrates and discusses a change in the conformation of PVA matrix and in turn a systematic variation in the morphology and patterning of in situ precipitated magnetite particles as a result of changing the total number of iron ions with respect to a constant number of hydroxyl functional groups of the polymer matrix. The observed variation in morphological feature reveals a close interplay between the inorganic-organic phase of the system to mould the matrix structure and to regulate the precipitation products.
Table 1 Variation of the number of total iron ions in $S_{1}, S_{2}$ and $S_{3}$.

\begin{tabular}{|c|c|c|c|c|c|}
\hline $\begin{array}{c}\text { Sample } \\
\text { No. }\end{array}$ & $\begin{array}{c}\text { Vol. of } \\
0.5 \text { mass } \% \\
\text { PVA soln. } \\
V_{\text {PVA }} \mathrm{cm}^{3}\end{array}$ & $\begin{array}{c}\text { Vol. of } \\
0.144 \\
\mathrm{~mol} / \mathrm{L} \\
T_{\mathrm{Fe}} \text { soln. } \\
V_{\mathrm{Fe}} \mathrm{cm}^{3}\end{array}$ & $\begin{array}{c}\text { Total } \\
\text { Vol. } \\
V_{\mathrm{PVA}}+V_{\mathrm{Fe}} \\
=\mathrm{V} \mathrm{cm}^{3}\end{array}$ & $\begin{array}{l}\text { Wt. of } \\
T_{\mathrm{Fe}} \text { in } \mathrm{V} \\
\mathrm{cm}^{3} \\
\mathrm{Wg}\end{array}$ & $\begin{array}{l}\text { No. of } T_{\mathrm{Fe}} \\
\text { in } \mathrm{Wg}\end{array}$ \\
\hline $\mathrm{S}_{1}$ & 20 & 5 & 25 & 0.617 & $5 \times 10^{21}$ \\
\hline $\mathrm{S}_{2}$ & 20 & 20 & 40 & 0.987 & $10 \times 10^{21}$ \\
\hline $\mathrm{S}_{3}$ & 20 & 160 & 180 & 4.44 & $40 \times 10^{21}$ \\
\hline
\end{tabular}

\section{Experimental Procedure}

Aqueous solution of polyvinyl alcohol (crystalline powder, average molecular weight 125,000) having concentration 0.5 mass $\%$ was prepared by dissolving the weighed amount of PVA in doubly de-ionized water at $80^{\circ} \mathrm{C} .300 \mathrm{ml}$ of $T_{\mathrm{Fe}}$ (total iron ion) solution containing both ferrous and ferric ions $\left(\left[\mathrm{Fe}^{3+}\right] /\left[\mathrm{Fe}^{2+}\right]=1.5\right)$ having concentration $0.144 \mathrm{~mol} / \mathrm{L}$ was prepared by dissolving $5.4 \mathrm{~g}$ of anhydrous ferric chloride $\left(\mathrm{FeCl}_{3}\right)$ and $2 \mathrm{~g}$ of ferrous chloride tetra hydrate $\left(\mathrm{FeCl}_{2} \cdot 4 \mathrm{H}_{2} \mathrm{O}\right)$ in requisite amount of deionised water. ${ }^{11)}$ Three different sets each comprising $20 \mathrm{ml}$ of the above PVA solution were mixed with different volumes of $T_{\mathrm{Fe}}$ solutions, such that $5 \times 10^{21}, 10 \times 10^{21}$ and $40 \times 10^{21}$ number of iron ions could be incorporated at room temperature, in the three sets $\left(\mathrm{S}_{1}, \mathrm{~S}_{2}, \mathrm{~S}_{3}\right)$ respectively, as shown in Table 1 . The $\mathrm{pH}$ of the resulting solution was 3 . Iron ion loaded PVA systems were kept in an oven for 48 hours at $(40 \pm 5)^{\circ} \mathrm{C}$ under nitrogen atmosphere. Gelation of PVA led to the formation of light orange coloured polymeric membrane in all the three sets. The iron ion loaded PVA membranes were soaked separately in $2.05 \mathrm{~mol} / \mathrm{L}$ sodium hydroxide solution at $40^{\circ} \mathrm{C}$ for 24 hours following which colour of the membranes were changed from orange to black as per the following equations:

$$
\begin{aligned}
\mathrm{FeCl}_{2}+2 \mathrm{FeCl}_{3}+8 \mathrm{NaOH} & \rightarrow \mathrm{Fe}(\mathrm{OH})_{2}+2 \mathrm{Fe}(\mathrm{OH})_{3}+8 \mathrm{NaCl} \\
\mathrm{Fe}(\mathrm{OH})_{2}+2 \mathrm{Fe}(\mathrm{OH})_{3} & \rightarrow \mathrm{Fe}_{3} \mathrm{O}_{4}+4 \mathrm{H}_{2} \mathrm{O}
\end{aligned}
$$


Next, the black coloured PVA membranes were washed thoroughly with doubly deionised water and dried in an oven at $50^{\circ} \mathrm{C}$ under nitrogen atmosphere. The dried specimens were characterized through X-ray diffractometry (XRD, PTS 3003, Seifert, Germany) and scanning electron microscopy (SEM, JSM 840 A, JEOL, Japan). The experiments were repeated several times to confirm the reproducibility.

In an environment of a fixed number of hydroxyl functional groups of PVA the kinetics of PVA-iron ion interaction is a function of a varying number of total iron ions. The number of hydroxyl functional groups in PVA corresponding to the average molecular weight of 125,000 has been calculated from the degree of polymerisation $\left(D_{\mathrm{p}}\right)$ using the formula, $M=D_{\mathrm{p}} \times m$, where $M$ is the average molecular weight of the polymer and $m$ is the molecular weight of the monomer unit. ${ }^{12}$ )

\section{Results and Discussion}

PVA is a crystalline, polar (atactic), hydrophilic and chemically heterogeneous polymer. Presence of a carbon chain backbone with strong covalent $\sigma$ bonds having bond length in the order of $0.1 \sim 0.15 \mathrm{~nm}$ is the main structural feature of PVA. Much weaker intermolecular forces interact between the monomer chains at a distance of $0.3 \sim 0.4 \mathrm{~nm}$. Gelation of PVA solution under optimum conditions leads to the formation of a noncovalent spatial network. The major types of intermolecular links in the junction knots are hydrogen bonding between hydroxyl groups of the neighbouring polymer chains. Syndiotactic sites in the chains are responsible for the formation of hydrogen bonds while the isotactic sites participate in the intermolecular interactions responsible for sterically entrapment of cations. ${ }^{10)}$ Induced by external factors like solvent interaction, applied stress or thermal energy, the polymer gels in general undergo conformational changes. This change does not involve any rupture of chemical bond. The internal rotation of the monomer chains around a single bond makes this phenomenon possible. A polymer chain may assume various conformations e.g., linear, rigid rod, helical, globular or coiling of the polymer chains under the influence of the above mentioned external factors. An absolutely flexible chain coils up because it decreases its surface area and free energy. The thermodynamic probability or the number of conformations that a polymer chain can assume is given by Gauss's formula. $^{13)}$

SEM studies all the three iron ion loaded PVA gel samples before the precipitation of iron oxide confirmed the formation of preorganized PVA structure. A PVA gel containing $5 \times$ $10^{21}$ iron ions revealed the parallel alignment of long PVA tubules as shown in Fig. 1(a). A two times change in the total number of iron ions to $10 \times 10^{21}$ in PVA gel did not bring any noticeable change in the matrix conformation. However, raising the number of total iron ions to $40 \times 10^{21}$ change the matrix conformation to a porous PVA network made by the coiling of the PVA tubules, as shown in Fig. 1(b).

Formation of the magnetite phase in all the three samples was confirmed by the X-ray diffraction pattern. An almost identical diffraction pattern was obtained from all the three
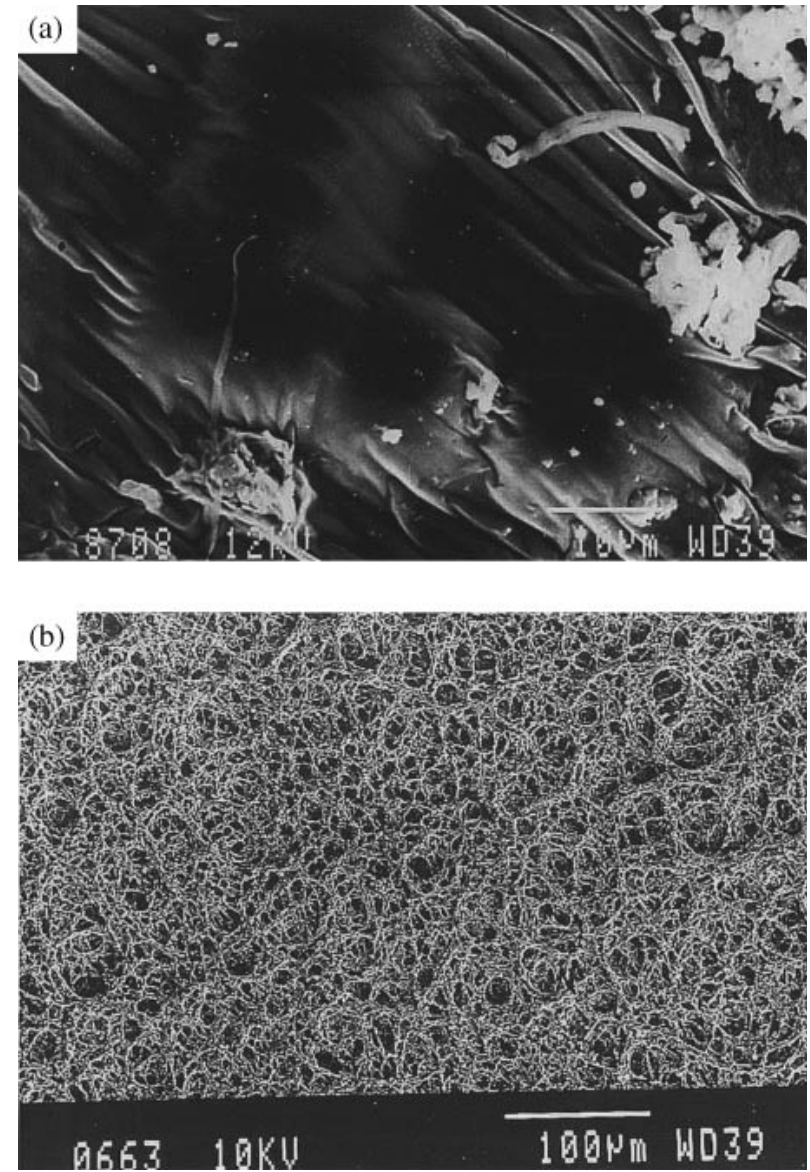

Fig. 1 (a) SEM micrograph of co aligned long tubules in iron ion $\left(5 \times 10^{21}\right)$ loaded PVA gel. (b) SEM micrograph of a porous network formed by coiling of the co aligned tubules in iron ion $\left(40 \times 10^{21}\right)$ loaded PVA gel.

samples. A representative XRD pattern comprising (220), (311), (400), (511) and (440) plane reflections of magnetite is shown in Fig. 2. No peak corresponding to maghemite or hematite could be noticed.

SEM study of sample $\mathrm{S}_{1}$ containing $5 \times 10^{21}$ iron ions revealed the growth of linearly arranged round shaped magnetite particles in the size range of $300-600 \mathrm{~nm}$ on the polymeric surface, as shown in Fig. 3(a). A change in the number of iron ions from $5 \times 10^{21}$ to $10 \times 10^{21}$ in sample $S_{2}$ manifested the formation of bead like structure of magnetite particles forming a two-dimensional array, as shown in Fig. 3(b). A careful observation of the microstructure revealed the apparent bead size was in the range of $500-600 \mathrm{~nm}$, form a uniform distribution on the PVA surface. It is interesting to note that the formation of beads is a result of mesoporous cluster formation of nanosized magnetite particles. Ultra-fine size particles of the cluster could not be resolved individually under SEM and the size could be estimated by Scherer's formula using full width at half maxima of (311) plane reflection of the diffraction pattern which indicated an average particle size of $80 \mathrm{~nm}$ forming the bead like the clusters. In sample $S_{3}$, an eight times increase in the total number of iron ions resulted in the formation of mesoporous coating of magnetite particles on the PVA surface, as shown in Fig. 3(c). The individual particle size in this case was 


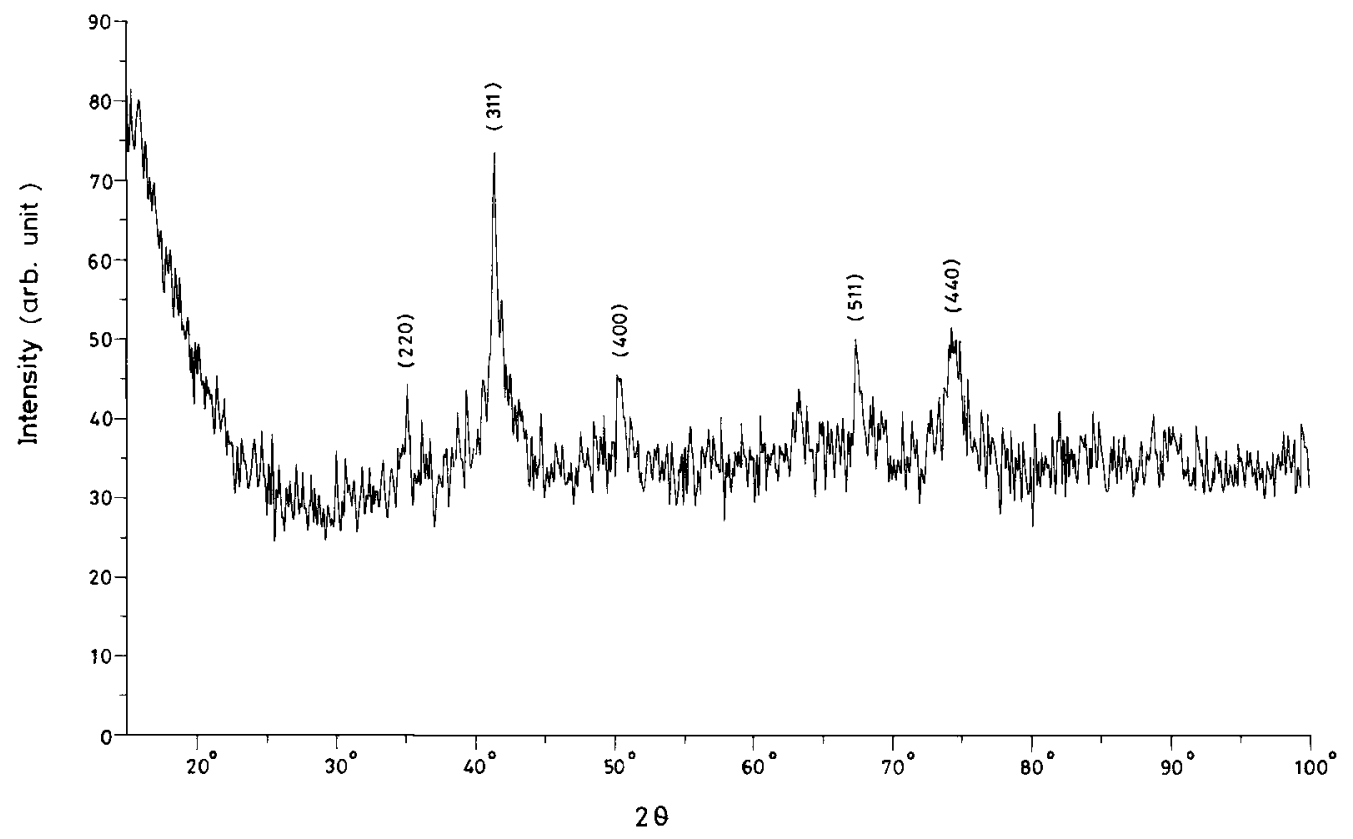

Fig. 2 X-ray diffraction pattern of sample $\mathrm{S}_{3}$.
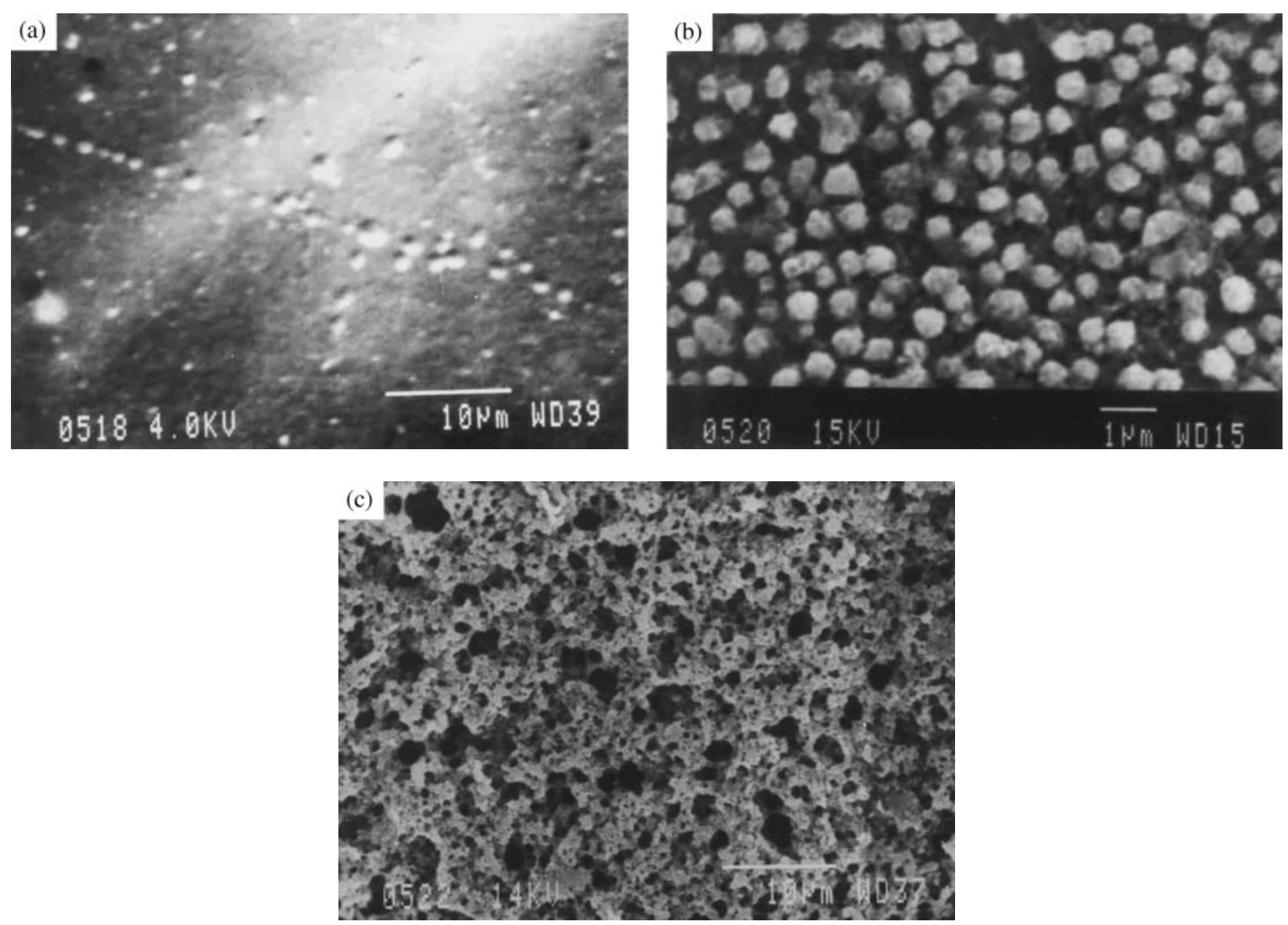

Fig. 3 (a) SEM micrograph of a pair of linear arrays of magnetite particles (300-600 nm) in sample $\mathrm{S}_{1}$. (b) SEM micrograph of uniformly distributed mesoporous beads of magnetite particles $(500-600 \mathrm{~nm})$ in sample $\mathrm{S}_{2}$. (c) SEM micrograph of uniform mesoporous coating of magnetite particles on the PVA surface in sample $S_{3}$. 
estimated to be in the range of 10-12 nm from the Bragg peak corresponding to (311) plane reflections of magnetite, using Scherer's formula.

As the precipitation of the inorganic substance in the polymer replicates the underlying structure of the matrix, formation of magnetite arrays in sample $S_{1}$ is in correspondence with a highly ordered polymeric conformation comprising co aligned PVA tubules. A change in the total number of iron ions by a factor of two did not lead to a major variation in the polymeric matrix and hence, due to a relatively higher nucleation rate at discrete precipitation sites, we could notice the precipitation of porous bead like particles forming a regular pattern on the PVA surface. The mesoporous structure of PVA matrix remained intact even after eight times increase in the total number of iron ions. However, a relatively higher number of iron ions lead to an enhanced nucleation and slow growth of magnetite particles. Finally, possessing a very high surface energy magnetite nanoparticles undergo self-diffusion and revealed a two dimensional epitaxial growth forming a mesoporous PVA-magnetite film.

The obtained results further generalise the hypothesis proposed by Lee et al. ${ }^{14)}$ that the amount of polymer and its molecular length can affect the distribution of cations and vice-versa.

\section{Conclusions}

The above results demonstrate an interesting observation in the morphological features of PVA-magnetite composite based on variation of the number of total iron ions with respect to a fixed number of hydroxyl functional groups of PVA. Three different numbers of total iron ions taken as
$5 \times 10^{21}, 10 \times 10^{21}$ and $40 \times 10^{21}$ had been experimented. It was observed that an exponential increase in metal cation numbers keeping the number of hydroxyl functional groups of PVA constant, showed an increase in the magnetite particle density, as well as, a variation in the morphology of the composite film ranging from a pair of highly ordered set of linear arrays of magnetite particles to the mesoporous coating films.

\section{REFERENCES}

1) S. Mann and G. A. Ozin: Nature 382 (1996) 313-318.

2) S. Mann, B. R. Heywood, S. Rajam and J. D. Birchall: Nature 334 (1988) 692-695

3) S. Mann, J. P. Hannington and R. J. P. William: Nature 324 (1986) 565-567.

4) A. Sinha, S. K. Das, B. R. Kumar, S. Chakraborty, V. Rao and P. Ramachandrarao: J. Mater. Synth. and Processing 8 (2000) 109-113.

5) P. Calvert: Materials Science and Technology, eds. R. W. Cahn, P. Hassen and E. J. Kramer, (VCH Verlagsgesellschaft mbH, P.O. Box 101161, D-69451 Weinheim, Germany, 1996) pp. 74-78.

6) D. Schuler: J. Mol. Microbio. Biotech. 1 (1999) 79-86.

7) M. Ali, H. Schnablegger and S. Mann: Nature 402 (1999) 393-395.

8) E. F. Desouza and F. Galembeek: J. Mater. Sci. 32 (1997) 2207.

9) S. Baskaran, L. Song, J. Liu, Y. L. Chen and G. L. Graff: J. Am. Ceram. Soc. 81 (1998) 401-408.

10) A. Sinha, J. Chakraborty, S. K. Das, S. Das, V. Rao and P. Ramachandrarao: Mater. Trans. 42 (2001) 1672-1675.

11) A. E. Regazzoni, G. A. Urrutia, M. A. Blesa and A. J. G. Maroto: J. Inorg. Nucl. Chem. 43 (1981) 1489-1493.

12) V. R. Gowariker, N. V. Viswanathan and J. Sridhar: Polymer Science, (New Age International Publishers, New Delhi, India, 1986) pp. 91-92.

13) A. Tager: Physical Chemistry of Polymers, (MIR Publishers, Moscow, 1978) pp. 100-102.

14) S. J. Lee, E. A. Benson and W. M. Kriven: J. Am. Ceram. Soc. 82 (1999) 2049-2055. 